\title{
Delegiertenversammlung segnet Detailkonzept zur Tarifrevision ab
}

\author{
Kerstin Schutz ${ }^{a}$, Christian Oeschger ${ }^{b}$ \\ ${ }^{a}$ Expertin, Abteilung Ambulante Versorgung und Tarife; ${ }^{b}$ Wissenschaftlicher Mitarbeiter, Abteilung Ambulante Versorgung und Tarife
}

In der Delegiertenversammlung (DV) der FMH vom 26. Januar 2017 hatten die Delegierten der Dachverbände, des VLSS und des VSAO sowie von Ärztinnen Schweiz unter anderem auch Beschlüsse zur Tarifrevision TARCO zu fassen. Indem die DV dem Detailkonzept, den Tarifierungsgrundsätzen sowie dem Konzept zu den Dignitäten zugestimmt hat, erteilt sie den weiteren Revisionsarbeiten «grünes Licht».

Seit der letzten Delegiertenversammlung der FMH (DV) vom 7. September 2016 haben die Mitarbeitenden der Abteilung Ambulante Versorgung und Tarife intensiv am Detailkonzept für das Projekt TARCO gearbeitet. Dieses Konzept wurde am 26. Januar 2017 zusammen mit den Tarifierungsgrundsätzen, dem Konzept zu den Dignitäten und weiteren Unterlagen der DV zur Genehmigung vorgelegt. Die Delegierten sind dem Vorschlag der FMH gefolgt und beauftragen damit das Departement Ambulante Versorgung und Tarife, die Arbeiten weiterzuführen.

\section{Wesentliche Änderungen in den Tarifie- rungsgrundsätzen}

Die Delegierten hatten neben dem Detailkonzept auch die überarbeiteten Tarifierungsgrundsätze zu genehmigen. Wichtig: Die FMH wird keine kostenneutrale Revision anstreben, die Tarifstruktur nicht normieren und sich im Projekt TARCO auf den praxisambulanten Bereich konzentrieren. Des Weiteren werden die ärztliche Unterstützung sowie die allgemeinen Leistungen im Operationssaal grundlegend überarbeitet und gemäss den Rückmeldungen aus der Ärzteschaft revidiert. Ausserdem sollen die geforderte Plausibilisierung der volumenstärksten Handlungsleistungen eines Kapitels umgesetzt und wo notwendig die einzelnen Positionen wieder mit einer Qualitativen Dignität versehen werden.

\section{Fokus auf praxisambulante Leistungen}

Einzelne Tarifpartner haben beschlossen, unabhängig von der FMH an einem eigenen Tarifvorschlag weiterzuarbeiten und dem Bundesrat im Oktober 2016 Vorschläge für die Anpassung des aktuellen Tarifs einge- reicht. Das bedeutet auch, dass die FMH nicht mehr über alle Daten verfügt, welche die Revisionspartner im Vorgängerprojekt eingebracht hatten. Der FMH liegen im ambulanten Bereich ausschliesslich Daten für die Arztpraxis vor, weshalb das Department vorgeschlagen hat, sich im Projekt TARCO vorwiegend auf den praxisambulanten Bereich zu fokussieren.

Der Fokus auf die praxisambulanten Leistungen bedeutet aber weder dass der Leistungskatalog stark beschnitten wird noch dass die FMH im Spital angestellte Ärztinnen und Ärzte nicht mehr vertritt.

1. Alle Leistungen, die in der freien Praxis erbracht werden, werden aus Praxissicht tarifiert. Nach ersten Schätzungen enthält die Tarifversion TARCO Vo.1 etwa 250 rein spitalambulante Leistungen, welche künftig nicht mehr im Projekt TARCO abgebildet werden, da die FMH zur Berechnung der technischen Leistung (TL) nicht mehr über die nötigen Daten verfügt. Die FMH wird sich bei diesen ebenso wie bei den praxisambulanten Leistungen aber auch weiterhin für die ärztliche Leistung ( $\mathrm{AL}$ ) einsetzen, welche mit demselben Referenzeinkommen hinterlegt sind.

2. Für die FMH sind alle ärztlichen Leistungen nach einem identischen Prinzip zu entschädigen - unabhängig davon, ob diese in der freien Praxis, im Spital oder durch einen Belegarzt erbracht werden. Die Entschädigung basiert dabei auf dem Referenzeinkommen sowie den entsprechenden Dignitätsfaktoren. Statt für die Tarifierung einzelner spitalambulanter Leistungen setzt sich die FMH im gesamten spitalambulanten Bereich für ein identisches Referenzeinkommen als Mindestansatz für die ärztliche Tätigkeit ein. 


\section{Plausibilisierung von Handlungsleistungen}

Die Plausibilisierung der Minutagen von volumenstarken Handlungsleistungen sowie die Überprüfung der korrekten Anwendung von Handlungs- oder Zeitleistungen sind Ziele des Projektes TARCO. Viele der FMH angeschlossenen Gesellschaften, das BAG und die Kostenträger fordern eine Plausibilisierung des Zeitbedarfs medizinischer Leistungen bei Handlungsleistungen.

In einem ersten Schritt wird der Schwerpunkt im Projekt TARCO auf die Plausibilisierung der Minutagen von volumenstarken Handlungsleistungen gelegt. Mit Unterstützung der Berner Fachhochschule (BFH) entsteht eine wissenschaftliche Studie, die gleichzeitig als Vorgehenskonzept dient. Der Aufwand soll für alle betroffenen Fachgesellschaften etwa gleich hoch sein. Die Teilnahme an diesem Projekt ist für die Gesellschaften allerdings freiwillig. Der Entwurf für das Projektkonzept wird aktuell durch die BFH bearbeitet. Über das definitive Konzept und das Vorgehen zur Plausibilisierung der BFH entscheidet die TARCO-Steuerungsgruppe («Cockpit»).

\section{Konzept Dignitäten}

Bereits gestartet ist die Arbeitsgruppe Dignitäten, die sich aus 18 Vertreterinnen und Vertretern der FMHDachverbände zusammensetzt. Die Arbeitsgruppe konnte in fünf Sitzungen drei neue Modelle zur Abbildung und zum Ersatz der heutigen quantitativen Dignitäten erarbeiten sowie Kriterien für die Anwendung der qualitativen Dignitäten definieren. Die Arbeitsgruppe hat die erarbeiteten Vorschläge einstimmig verabschiedet und diese mit einem entsprechenden Antrag der DV am 26. Januar 2017 vorgelegt. Dabei lassen sich die Elemente der quantitativen Dignitäten, die in den kommenden Monaten im Detail ausgearbeitet werden müssen, wie folgt zusammenfassen: Individuelle Arztdignität (IAD): Die individuelle ärztliche Dignität (IAD) soll das Lebenseinkommen der Ärzteschaft ausgleichen, das je nach Facharzttitel und
Schwerpunkt aufgrund der Weiterbildungszeiten unterschiedlich sein kann. Betrachtet wird lediglich das Einkommen, welches aus Tätigkeiten aus dem ambulanten Arzttarif generiert werden kann. Entscheidend dafür sind die Weiterbildungsprogramme des SIWF. Die IAD ist arztgebunden und kann für sämtliche Tarifpositionen auf die ärztliche Leistung angewendet werden. Effizienzzuschlag bei Zeitleistungen: Bei Handlungsleistungen ist zum Teil ein Effizienzgewinn möglich, wenn der Arzt weniger Zeit fürs Erbringen einer Leistung benötigt als tarifiert ist. Fachrichtungen, die vornehmlich mit Zeitleistungen abrechnen, können von dieser Effizienzsteigerung im aktuellen TARMED 01.08.00_BR aber nicht profitieren: Wenn diese Ärztinnen und Ärzte einen Patienten aufgrund ihrer Erfahrung effizienter behandeln, rechnen sie entsprechend weniger Zeitleistungen ab. Ein Zuschlag auf Zeitleistungen $a b$ einer gewissen Erfahrungsstufe soll diesem Umstand Rechnung tragen. Dieser Zuschlag ist arztgebunden und kann für sämtliche Zeitleistungen-Tarifpositionen auf die ärztliche Leistung angewendet werden. Leistungsbezogener Qualitätsfaktor LQF: Der LQF ist ein innovatives Instrument, um in Zukunft allfällige zusätzliche finanzielle Mittel aufgrund von festgelegten Qualitätskriterien der Fachgesellschaften innerhalb der einzelnen Kapitel zu verteilen. Der Faktor ist SAQM-basiert und ist leistungsgebunden.

\section{Weiterer Fahrplan}

Die Projektmitarbeitenden haben bereits Anfang 2017 mit den ersten Fachgesellschaften Kontakt aufgenommen und begonnen, erste Kick-off-Termine für die Arbeitsgruppen zu vereinbaren. Sofern die Fachgesellschaften den Zusammenarbeitsvertrag unterschrieben und retourniert haben, können die Arbeiten aufgenommen werden. Der weitere Zeitplan sieht dann wie folgt aus (siehe Tabelle am Seitenende).

Weitere Informationen finden Sie auf der Webseite der FMH unter www.fmh.ch $\rightarrow$ Ambulante Tarife sowie im Infoletter des Büro Tarifs (www.fmh.ch $\rightarrow$ Ambulante Tarife $\rightarrow$ Publikationen $\rightarrow$ Infoletter).
Korrespondenz: FMH / Abteilung Ambulante Versorgung und Tarife

Frohburgstrasse 15

CH-4600 Olten

Tel. 0313591230

Fax 0313591238

tarife.ambulant[at]fmh.ch

\begin{tabular}{ll}
\hline Zeitraum & Was \\
\hline 26.01 .2017 & Entscheid Detailkonzept \\
\hline $01.02 .-31.10 .2017$ & Inhaltliche Arbeiten in den Arbeitsgruppen \\
\hline $01.01 .-31.01 .2018$ & Vernehmlassungsphase (Fokus Qualitative Dignitäten) \\
\hline 22.03 .2018 & Delegiertenversammlung der FMH: Entscheidung über neue Tarifstruktur \\
\hline 03.05 .2018 & Ärztekammer der FMH: Entscheidung über neue Tarifstruktur und gegebenenfalls Urabstimmung \\
\hline 30.06 .2018 & Übergabe des Projekt-Ergebnisses an die Delegiertenversammlung der FMH \\
\hline 2018 & Schlussbereinigung mit den Tarifpartnern und Eingabe beim Bundesrat \\
\hline
\end{tabular}

